\title{
Xây dựng và thẩm định phương pháp phân tích hàm lượng một số oligosaccharide từ sữa me (HMOs) trong thực phẩm bổ sung bằng kỹ thuật sắc ký loỏng khối phổ (LC-MS/MS)
}

\author{
Nguyễn Thị Hồng Ngọc ${ }^{1^{*}}$, Mạc Thị Thanh Hoa ${ }^{1}$, Trần Hùng Sơn ${ }^{1}$, Ngô Mạnh Dũng ${ }^{2}$ \\ Cao Công Khánh ${ }^{1}$, Vũ Thị Thanh An ${ }^{1}$, Phạm Thị Thanh Hà, Lê Thị Hồng Hảo \\ ${ }^{1}$ Viện Kiểm nghiệm an toàn vệ sinh thực phẩm Quốc gia, Hà Nội, Việt Nam \\ ${ }^{2}$ Truiơng Đại học Dược Hà Nội, Việt Nam
}

(Ngày đến tòa soạn: 25/12/2020; Ngày chấp nhận đăng: 19/3/2021)

\section{Tóm tắt}

Kỹ thuật sắc ký lỏng khối phổ hai lần đã được ứng dụng nhằm nghiên cứu xây dựng và thẩm định phương pháp xác định hàm lượng một số oligosaccharide chính từ sữa mẹ (Human Milk Oligosaccharides - HMOs) gồm 2'-Fucosyllactose (2'-FL), Lacto-N-neotetraose (LNnT), Lacto-N-tetraose (LNT), 3'-Siallylactose (3'-SL) và 6'-Siallylactose (6'-SL) trong thực phẩm bổ sung dạng bột và dạng lỏng. Hệ pha động sử dụng gồm 2 kênh: kênh $\mathrm{A}$ (dung dịch acid formic $0,1 \%)$ và kênh $B$ (acetonitril) kết nối với cột phân tích HILIC $(1,7 \mu \mathrm{m} ; 2,1 \times 150 \mathrm{~mm})$ thông qua detector khối phổ cho thời gian phân tích ngắn, trong 10 phút có thể xác định đồng thời được cả 05 chất thuộc nhóm HMOs. Phương pháp đã được thẩm định cho thấy độ chính xác cao, nhanh, hiệu quả và đã được áp dụng vào phân tích các mẫu trên thị trường cho thấy khả năng ứng dụng cao trong thực tế. Giới hạn phát hiện và giới hạn định lượng cho cả 5 chất 2'-FL, LNnT, LNT, 3'-SL, 6'-SL tương ứng là $4,0 \mathrm{mg} / \mathrm{kg}$ và $10,0 \mathrm{mg} / \mathrm{kg}$. Khoảng tuyến tính của phương pháp nằm trong khoảng từ $0,4-40 \mathrm{Mg} / \mathrm{mL}$. Các thông số thẩm định khác bao gồm độ đúng $(\mathrm{R} \%$ 98,8 - 103 \%); độ chính xác (RSD\% 1,69 - 5,54 \%) đều đáp ứng được theo yêu cầu của AOAC. Quy trình được áp dụng vào thực tế phân tích 25 mẫu thực phẩm bổ sung (TPBS) trên thị trường cho kết quả tổng các HMOs trong mẫu TPBS dạng bột khoảng $0,1-0,3 \mathrm{~g} / 100 \mathrm{~g}$ và đối với TPBS dạng lỏng khoảng 0,01 - 0,03 g/100mL.

Tư khóa: 2'-Fucosyllactose (2'-FL), Lacto-N-neotetraose (LNnT), Lacto-N-tetraose (LNT), 3'-Siallylactose (3'-SL), 6'-Siallylactose (6'-SL); HMOs; Thực phâm bổ sung; HPLC; LC-MS/MS.

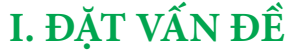

Oligosaccharide từ sũa mẹ (Human Milk Oligosaccharide - HMOs) là nhóm sinh khối dôi dào thứ ba sau đường sữa và lipid, đạt từ 5 đến $20 \mathrm{~g} / \mathrm{L}$ trong sũa mẹ [1]. Có tới $1 \% \mathrm{HMOs}$ được hấp thu ở đường tiêu hóa và được tìm thấy trong tuần hoàn toàn thân. Sự đa dạng và phong phú này chỉ có ở người và không thấy ở các động vật có vú khác. Hàm lượng HMOs phụ thuộc vào nhiều yếu tố khác nhau: giai đoạn tiết sũa, chế độ dinh dưỡng của người mẹ, khuynh hướng di truyền hoặc thậm chí là khu vực địa lý - thổ nhưỡng và môi trường kinh tế xã hội. Mặc dù HMOs được cấu tạo chỉ từ 05 loại monosacaride cơ bản, nhưng độ phức tạp về cấu trúc của $\mathrm{HMO}_{\mathrm{S}}$ trong sữa mẹ là rất phong phú và đa dạng. Hiện nay, hơn 200 loại oligosaccharide khác nhau về mặt cấu trúc đã được tìm thấy, trong đó có 19 chất thuộc nhóm HMOs đã được mô tả và 
xác định cấu trúc. Năm loại monosaccharide cơ bản hình thành cấu trúc HMOs gồm có glucose (Glc), galactose (Gal), N-Acetyl-Glucosamine (GlcNAc), furcose (Fuc) và acid sialic (Neu5Ac) [2-3] như trong Hình 1. Dựa trên cấu trúc cấu tạo, HMOs được xem như một prebiotic với các tác dụng chính như kháng khuẩn, kháng virus, kháng viêm. Tác dụng của HMOs đối với sức khỏe và sự phát triển của trẻ nhỏ được mô tả tổng quát như hoạt tính của một prebiotic, hoạt tính kháng khuẩn, kháng virus, hỗ trợ sự phát triển đường tiêu hóa... [3-4]. Do đó, hiện nay trên thị trường đã xuất hiện rất nhiều các sản phẩm được bổ sung một hoặc một số chất thuộc nhóm HMOs trong công thức bào chế. Tuy nhiên, tại Việt Nam hiện nay vẫn chưa có phương pháp phân tích xác định hàm lượng các HMOs được bổ sung vào các sản phẩm thực phẩm bổ sung. Vì vậy, việc phát triển một phương pháp phân tích để kiểm soát các sản phẩm này trên thị trường là điều cân thiết.

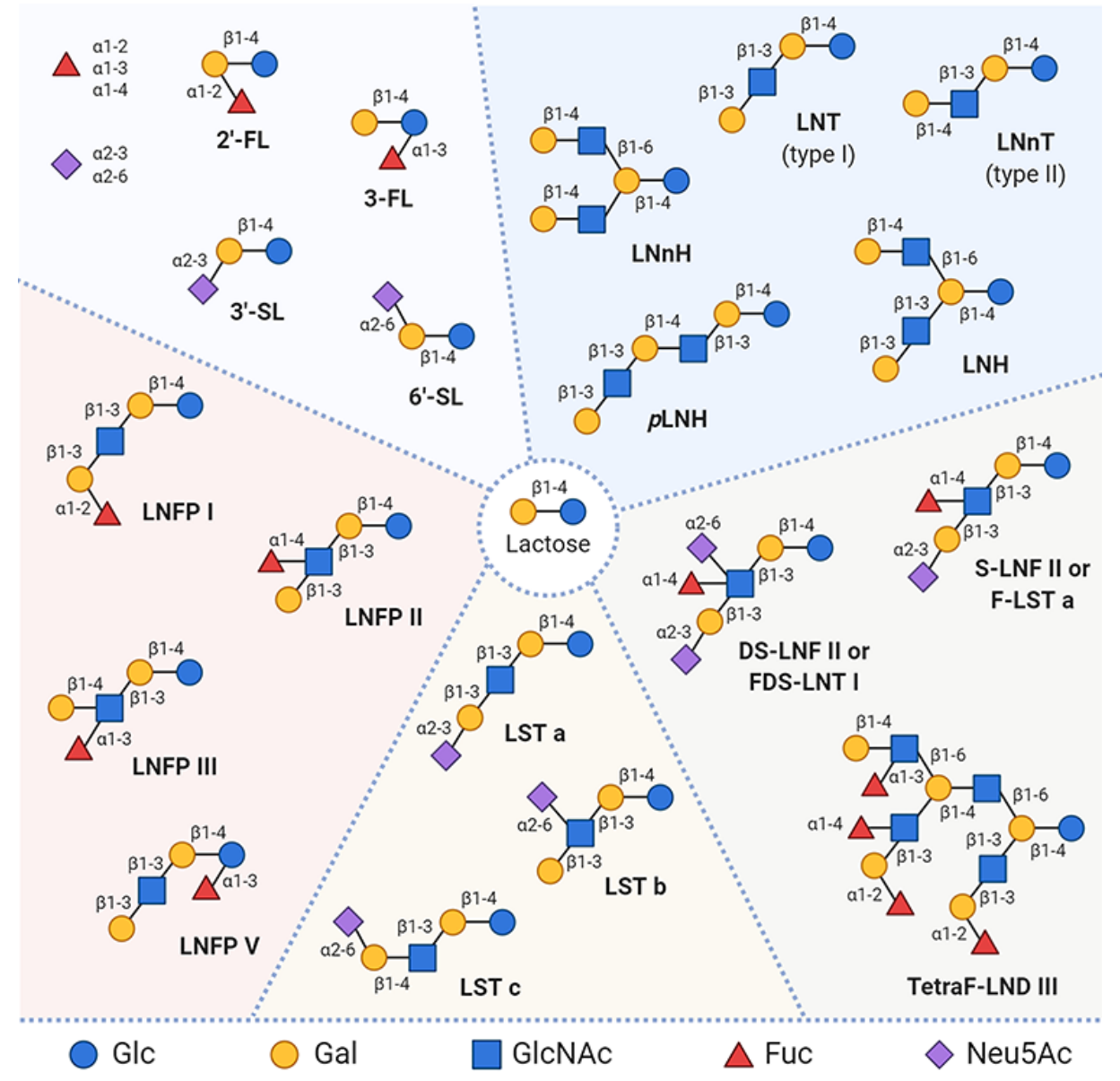

Hình 1. Liên kết hình thành HMOs tü 05 monosaccharide cơ bản [2]

Cho đến nay, đã có nhiều nghiên cứu xác định các chất thuộc nhóm HMOs trong các nền mẫu khác nhau với các kỹ thuật cũng rất đa dạng. Các các kỹ thuật có thể liệt kê như: kỹ thuật sắc ký bản mỏng (TLC), đo góc quay cực, sắc ký lỏng kết hợp với nhiều loại detector khác nhau [10-12]. Trong đó kỹ thuật sắc ký bản mỏng và đo góc quay cực chủ yếu được sử dụng để xác định độ tinh khiết của nguyên liệu, không áp dụng cho các dạng thành phẩm và bán thành phẩm. Kỹ thuật sắc ký lỏng với nhiêu loại detector khác nhau đã và đang được sử dụng rộng rãi để xác định các thành phần trong thành phẩm và bán thành phẩm. Các hình thức phát hiện và định lượng cũng đa dạng từ phân tích trực tiếp bằng RI [5], ELSD [6], HPAEC [7-9] MS/MS 
[10] đến phân tích gián tiếp bằng PDA và FLD [7].

Các phương pháp nêu trên đều sử dụng hóa chất và thiết bị thông thường để tiến hành phân tích, hâu hết phù hợp với điều kiện của các phòng thí nghiệm tại Việt Nam hiện nay. Phương pháp HPLC với detector FLD tuy đơn giản và có khả năng ứng dụng rộng nhửng trong quá trình phân tích cần sử dụng dẫn xuất tạo huỳnh quang, do vậy sẽ có thêm phần sai số ảnh hưởng do hiệu suất dẫn xuất. Trong khi đó, phương pháp sắc ký lỏng khối phổ hai lần là phương pháp cho độ nhạy tốt nhất, đồng thời cũng có triển vọng để phát triển phân tích đông thời nhiều chất nhất trong nhóm HMOs và có thể trực tiếp phân tích mà không cân thêm bước dẫn xuất.

Nghiên cứu này tập trung vào việc tối ưu hóa quá trình phân tích 05 chất thuộc nhóm HMOs gồm Glc, Gal, GlcNAc, Fuc và Neu5Ac trên thiết bị LC-MS/MS. Trong đó, các dung môi chiết và một số bước làm sạch được khảo sát để loại bỏ tối đa ảnh hưởng của nền mẫu đến quá trình phân tích. Các điều kiện phân tích được tối ưu hóa tự động trên thiết bị LC-MS/MS để thu được dữ liệu về mảnh phổ phân tích. Phương pháp sẽ được thẩm định để xác nhận giá trị sử dụng và được áp dụng thử nghiệm thực tế để phân tích các mẫu TPBS thu mua trên thị trường.

\section{VÂTT LIỆU VÀ PHƯƠNG PHÁP NGHIÊN CỨU}

\subsection{Thiết bị, dụng cụ và hóa chất}

\subsubsection{Thiết bị và dưng cu}

Thiết bị chính sử dụng trong quá trình phân tích là hệ thống sắc ký lỏng Acquity kết nối với khối phổ 3 tứ cực Xevo TQD của hang Waters, Mỹ. Cột phân tích mẫu là cột XBridge HILIC $(3,5 \mu \mathrm{m}, 2,1 \mathrm{~mm} \times 150 \mathrm{~mm})$ bản chất silica và cột BEH Glycan Amide $(1,7 \mu \mathrm{m}, 2,1 \times 150 \mathrm{~mm})$. Ngoài ra, nghiên cứu còn sử dụng một số thiết bị phụ trợ và dụng cụ thông thường khác trong phòng thí nghiệm.

\subsubsection{Hóa chất, chất chuân:}

Các hóa chất sử dụng trong nghiên cứu đều thuộc loại tinh khiết phân tích, bao gồm: Chuẩn 2'- fucosyllactose, lacto- $\mathrm{N}$-tetraose, lacto- $\mathrm{N}$-neotetraose, 3'-sialylactose, 6'-sialylactose của Carbosynth với độ tinh khiết $\geq 98,0 \%$. Một số hóa chất khác được sử dụng trong quá trình phân tích và xứ lý mẫu: Nước cất hai lần khử ion, methanol, chloroform, acetonitri, bột làm sạch $\mathrm{C}_{18}$, bột carbon graphit - Agilent, $\mathrm{Mỹ}, \mathrm{K}_{4} \mathrm{Fe}(\mathrm{CN})_{6} \cdot 3 \mathrm{H}_{2} \mathrm{O}$ (tinh khiết phân tích), $\mathrm{Zn}\left(\mathrm{CH}_{3} \mathrm{COO}\right)_{2}$ (tinh khiết phân tích), acid trichloroacetat (tinh khiết phân tích).

\subsection{Mẫu nghiên cứu}

- Đối tượng nghiên cứu: 25 thực phẩm bổ sung (TPBS) có chứa HMOs được lấy ngẫu nhiên trên thị trường Hà Nội, cụ thể bao gồm 15 mẫu TPBS dạng bột và 10 mẫu TPBS dạng lỏng.

- Lựa chọn mẫu trắng: áp dụng quy trình phân tích đã lựa chọn và tiến hành phân tích một số mẫu TPBS không chứa thành phần cung cấp HMOs. Chọn mẫu trắng là mẫu không có tín hiệu trùng với thời gian lưu của chất phân tích trong mẫu chuẩn.

\subsection{Phương pháp nghiên cứu}

\subsubsection{Khảo sát và xây dựng quy trình phân tích}

* Khảo sát và tối ưu hóa quá trình phân tích trên khối phổ 
- Để tối ưu hóa điều kiện phân tích trên khối phổ, các dung dịch chuẩn 2'-FL, LNT, LNnN, 3'- SL, 6'-SL được chuẩn bị ở nông độ $5 \mu \mathrm{g} / \mathrm{mLvà} \mathrm{tiến} \mathrm{hành} \mathrm{tối} \mathrm{ưu} \mathrm{hóa} \mathrm{tự} \mathrm{động} \mathrm{trên} \mathrm{thiết} \mathrm{bị} \mathrm{Xevo}$ TQD của hãng Waters để thu được các điều kiện về mảnh phổ của chất phân tích.

- Đối với quá trình tách sắc ký, 2 loại cột được lựa chọn khảo sát gồm: Cột BEH Glycan Amide $(1,7 \mu \mathrm{m}, 2,1 \times 150 \mathrm{~mm})$ và cột HILIC $(3,5 \mu \mathrm{m}, 2,1 \times 150 \mathrm{~mm})$ bản chất silica, dung dịch chuẩn hỗn hợp gồm cả 5 chất 2'-FL, LNT, LNnN, 3'- SL, 6'-SL ở nồng độ $1 \mu \mathrm{g} / \mathrm{mL}$ được phân tích với cùng hệ pha động bao gồm kênh $\mathrm{A}$ : acid formic $0,1 \%$ và kênh $\mathrm{B}$ : acetonitril.

* Khảo sát quy trinh chiết và làm sạch mẫu:

Mẫu sau khi được cân với lượng thích hợp $(2-5 \mathrm{~g})$ vào các ống ly tâm $50 \mathrm{~mL}$, thêm chuẩn ở mức nông độ $4 \mu \mathrm{g} / \mathrm{mL}$, bổ sung thêm $40 \mathrm{~mL}$ các dung môi và thuốc thử chiết mẫu; 4 hệ dung môi và thuốc thử khảo sát bao gồm: (1) thuốc thử Cazzer, (2) acid trichloroacetat $1 \%$ trong nước, (3) hệ dung môi chloroform : methanol (3:1, v/v), (4) dung môi methanol 100\%. Sau khi chiết, mẫu được ly tâm và định mức vừa đủ $50 \mathrm{~mL}$ bằng các dịch chiết và phân tích trên LC-MS/ MS để đánh giá độ thu hồi.

Đối với quy trình làm sạch, sau khi lựa chọn được dung môi chiết thích hợp, hút chính xác 1,0 $\mathrm{mL}$ dịch chiết khảo sát làm sạch bằng 2 loại bột $\mathrm{SPE}$ khác nhau: bột carbon graphit và bột $\mathrm{C}_{18}$. Ly tâm 13.000 vòng/phút, lấy phân dịch trong, phân tích trên LC-MS/MS và đánh giá độ thu hổi.

\subsubsection{Thâm định phương pháp}

Phương pháp được thẩm định theo đúng yêu cầu của $\mathrm{AOAC}$ với các thông số trên cả 2 nền mẫu TPBS dạng lỏng và dạng bột, bao gồm độ đặc hiệu, khoảng tuyến tính, độ lặp lại, độ thu hôii, giới hạn phát hiện và giới hạn định lượng.

\subsubsection{Phân tích mẫu thực tế}

Sau quá trình khảo sát điều kiện phân tích, quy trình được áp dụng để phân tích 5 chất HMOs trong 25 mẫu TPBS thu thập được.

\section{KẾT QUẢ VÀ BÀN LUẬN}

Dựa trên một số tài liệu tham khảo [5-8] và tính chất phân cực của nhóm HMOs [1], 02 loại cột HILIC và cột Amide đã được lựa chọn để khảo sát điều kiện tách trên sắc ký lỏng của các chất phân tích. Ngoài ra, do nền mẫu là TPBS dạng lỏng và dạng bột thường chứa hàm lượng lớn protein, lipid, vitamin, khoáng chất,... bước chiết và làm sạch mẫu trước khi phân tích trên LC-MS/MS là cân thiết.

\subsection{Khảo sát điều kiện sắc ký}

Qua tham khảo các tài liệu [7-8], [10] kết hợp với tiến hành khảo sát, điều kiện sắc ký để xác định đông thời HMOs được lựa chọn như sau:

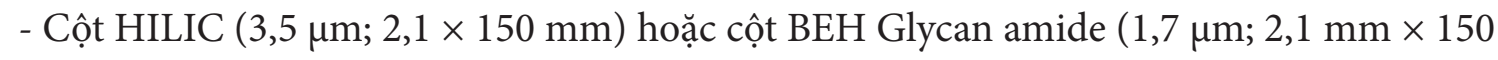
$\mathrm{mm})$ trước cột bảo vệ amide $\mathrm{BEH}(1,7 \mu \mathrm{m} ; 2,1 \mathrm{~mm} \times 50 \mathrm{~mm})$.

- Pha động: acetonitril: acid formic.

- Tốc độ dòng: 1,0 mL/phút. 
- Thể tích tiêm mẫu: $10 \mu \mathrm{L}$.

- Chương trình gradient (như ở Bảng 1).

Bảng 1. Chương trìn gradient phân tích HMOs

\begin{tabular}{ccc}
\hline Thòi gian (min) & Kênh A (acid formic 0,1\%) & Kênh B (acetonitril) \\
\hline 0,0 & 40 & 60 \\
2,0 & 40 & 60 \\
2,5 & 80 & 20 \\
7,5 & 80 & 20 \\
8,0 & 40 & 60 \\
10,0 & 40 & 60 \\
\hline
\end{tabular}

Kết quả sắc ký khảo sát khả năng phân tách các chất phân tích khi sử dụng cột HILIC và Amide được thể hiện trong Hình 2. Kết quả cho thấy, sắc ký đồ tổng (TIC) của dung dịch chuẩn phân tích trên cột HILIC cho thời gian lưu, thời gian phân tích cũng như khả năng tách tốt hơn so với cột amide.

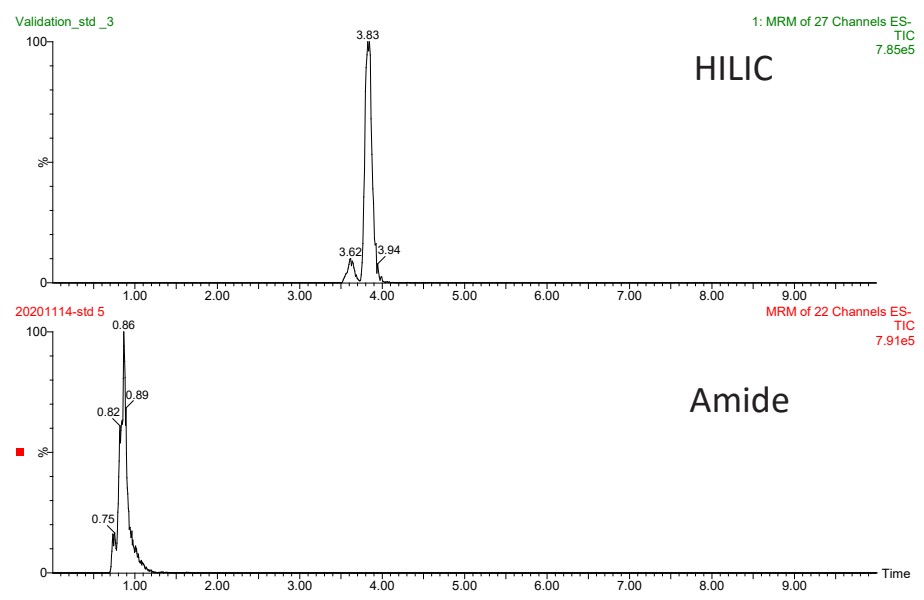

Hinh 2. Sắc ký đồ tông TIC dung dịch chuân hỗn hợp 5 chất 2'-FL, LNT, $L N n N$, 3'- SL, 6'-SL nồng độ $4 \mu \mathrm{g} / \mathrm{mL}$

\subsection{Khảo sát điều kiện chiết mẫu}

Các mẫu trắng được thêm chuẩn hỗn hợp 05 chất gồm: 2'-FL, LNT, LNnN, 3'- SL, 6'-SL ở nồng độ $4 \mu \mathrm{g} / \mathrm{mL}$. Sau đó tiến hành khảo sát điều kiện chiết mẫu sử dụng 04 dung dịch chiết khảo sát gồm: thuốc thử Carrez, dung dịch TCA $1 \%$, methanol, $\mathrm{CHCl}_{3:} \mathrm{MeOH}(3: 1, \mathrm{v} / \mathrm{v})$. Kết quả độ thu hôi được trình bày trong Bảng 2.

Bảng 2. Kết quả hiệu suất thu hồi sau quy trinh kháo sát dung môi chiết mẫu

\begin{tabular}{cccccc}
\hline Dung $\boldsymbol{m o ̂} \boldsymbol{i}$ & $\mathbf{2 - F L}$ & $\mathbf{L N T}$ & $\mathbf{L N n T}$ & $\mathbf{3 - S L}$ & 6-SL \\
\hline Carrez & $84,7 \%$ & $87,7 \%$ & $93,9 \%$ & $94,3 \%$ & $94,9 \%$ \\
$\mathbf{T C A} \mathbf{1 \%}$ & $95,5 \%$ & $93,5 \%$ & $99,1 \%$ & $91,0 \%$ & $92,7 \%$ \\
$\mathbf{H C l}_{3}: \mathbf{M e O H}$ & $99,0 \%$ & $98,8 \%$ & $98,0 \%$ & $98,8 \%$ & $99,6 \%$ \\
$\mathbf{M e O H}$ & $88,5 \%$ & $93,7 \%$ & $90,1 \%$ & $99,4 \%$ & $92,1 \%$ \\
\hline
\end{tabular}


Bảng 2 cho thấy với dung môi là hỗn hợp $\mathrm{CHCl}_{3}: \mathrm{MeOH}(3: 1, \mathrm{v} / \mathrm{v})$ hiệu suất thu hồi là cao nhất nên hỗn hợp dung môi $\mathrm{CHCl}_{3}: \mathrm{MeOH}(3: 1, \mathrm{v} / \mathrm{v})$ đã được lựa chọn cho các bước khảo sát tiếp theo.

\subsection{Khảo sát điều kiện làm sạch}

Các dịch chiết mẫu thu được sau quá trình xử lý với hỗn hợp $\mathrm{CHCl}_{3}: \mathrm{MeOH}(3: 1, \mathrm{v} / \mathrm{v})$ tiếp tục làm sạch bằng $0,15 \mathrm{~g}$ bột $\mathrm{C}_{18}$ và $0,15 \mathrm{~g}$ than hoạt tính để loại bỏ các thành phần còn lại như vi khoáng, chất tạo hương, chất tạo màu,... Kết quả thu được như Hình 3.

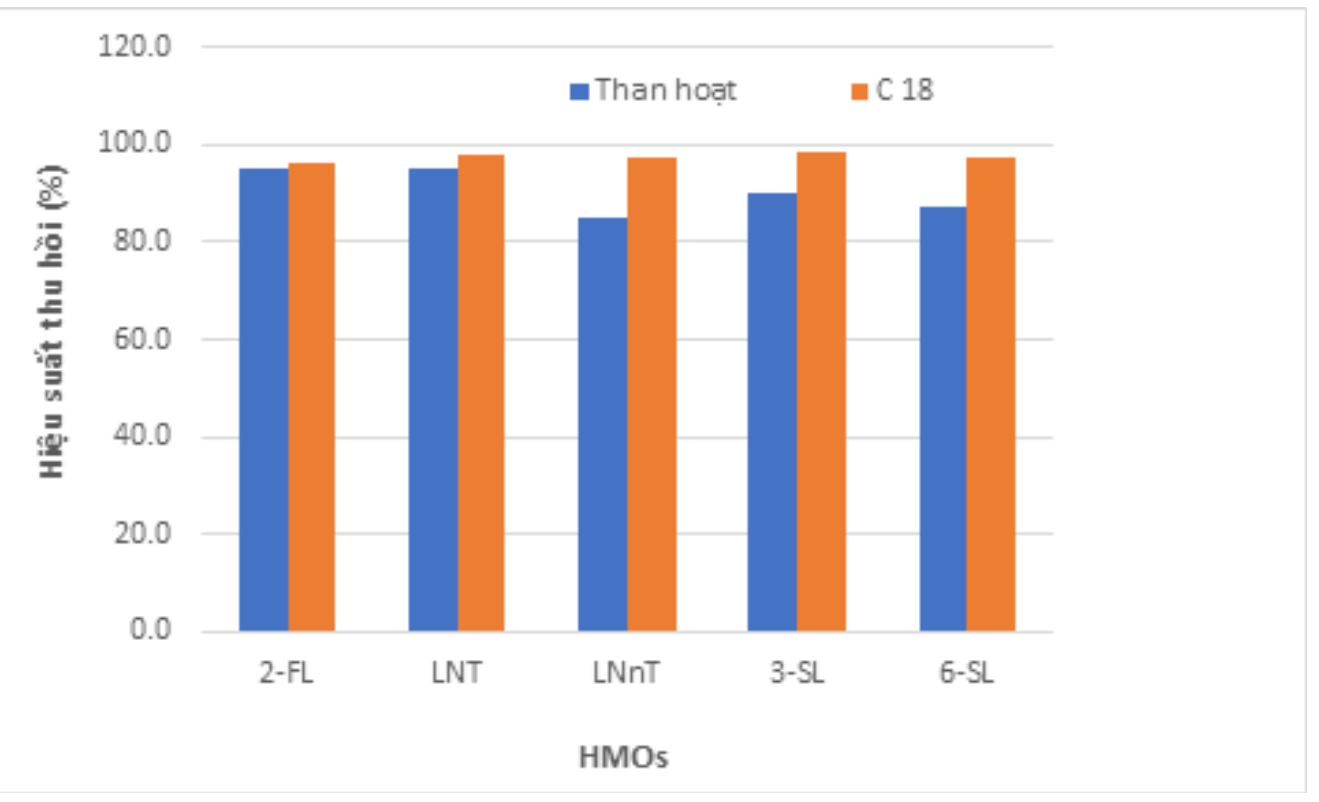

Hình 3. Kết quả hiệu suất thu hồi sau quá trinh làm sạch

Kết quả khảo sát ở Hình 3 cho thấy, sau khi làm sạch với bột than hoạt tính, hiệu suất thu hôi giảm xuống đồng thời dịch làm sạch vẫn còn màu so với các mẫu làm sạch bằng bột $\mathrm{C}_{18}$. Chính vì thế, bột làm sạch $\mathrm{C}_{18}$ dã được lựa chọn để làm sạch sau quá trình tách chiết trước khi tiến hành phân tích trên thiết bị LC-MS/MS.

Như vậy, sau các bước khảo sát, quy trình phần tích một số HMOs (2'-FL, LNT, LNnT, 3’SL, 6'SL) gồm các bước chính như sau: cân chính xác 2-5 g mẫu vào các ống ly tâm $50 \mathrm{~mL}$, thêm khoảng $35 \mathrm{~mL}$ dung môi chiết $\mathrm{CHCl}_{3}: \mathrm{MeOH}(3: 1, \mathrm{v} / \mathrm{v})$, lắc ngang trong 30 phút; Ly tâm lấy dịch trong, định mức vừa đủ $50 \mathrm{~mL}$; lắc đều và lọc qua giấy lọc thu dịch lọc; hút chính xác $1 \mathrm{~mL}$ dịch lọc vào các ống $\mathrm{d}-\mathrm{SPE}$ chứa sẵn $0,15 \mathrm{~g}$ bột làm sạch $\mathrm{C}_{18}$, lắc vortex trong 1 phút, ly tâm 13.000 vòng/phút trong 2 phút; thu dịch trong vào lọ đựng mẫu và phân tích trên thiết bị LC-MS/MS.

\subsection{Xác nhận giá trị sử dụng của phương pháp phân tích}

\subsection{1. Đồ đăc hiệu}

Tiến hành phân tích theo các điều kiện sắc ký đã lựa chọn với mẫu dung dịch chuẩn nông độ $4 \mu \mathrm{g} / \mathrm{mL}$, mẫu trắng và mẫu trắng có thêm chuẩn. Kết quả thu được cho thấy, thời gian lưu của 2'-FL, LNT, LNnT, 3'-SL, 6'SL trong mẫu thêm chuẩn giống với trong mẫu chuẩn, trên sắc đồ mẫu trắng không xuất hiện píc trong khoảng thời gian lưu của chất phân tích trên mẫu chuẩn như trong Hình 4. Dựa trên số mảnh mẹ và mảnh con của các chất phân tích, số điểm IP thu được như trong Bảng 3. 


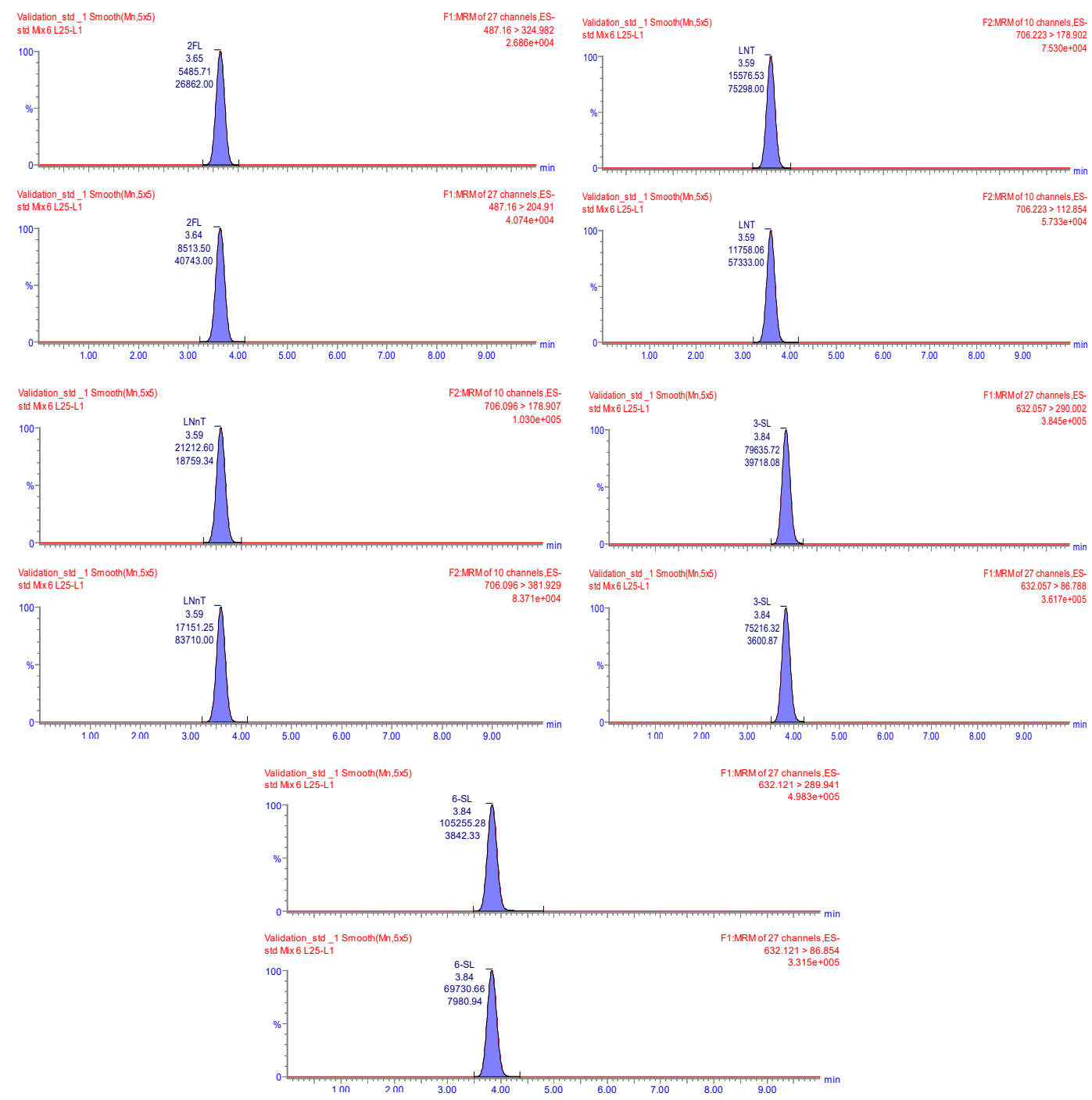

Hinh 4. Một số sắc kí đồ kết quả đánh giá độ đặc hiệu của 2'-FL, LNT, LNnT, 3'-SL, 6’SL

Bảng 3. Độ đặc hiệu của 2'-FL, LNT, LNnT, 3'-SL, 6'SL

\begin{tabular}{|c|c|c|c|c|c|c|}
\hline $\begin{array}{c}\text { Chất phân } \\
\text { tích }\end{array}$ & Mảnh mẹ & Mảnh con & Số điểm IP & $\begin{array}{c}\text { Tỷ lệ ion } \\
\text { trong mẫu } \\
\text { thêm chuân }\end{array}$ & $\begin{array}{c}\text { Tỷ lẹ ion } \\
\text { trong mẫu } \\
\text { chuẩn }\end{array}$ & Kết quả \\
\hline \multirow{2}{*}{$2^{\prime}-F L$} & 487,2 & $325,0^{*}$ & \multirow{2}{*}{4} & \multirow[t]{2}{*}{0,57} & \multirow[t]{2}{*}{0,52} & \multirow{2}{*}{ Đạt } \\
\hline & 487,2 & 205,0 & & & & \\
\hline \multirow{2}{*}{$L N T$} & 706,2 & $178,9^{*}$ & \multirow{2}{*}{4} & \multirow[t]{2}{*}{0,82} & \multirow[t]{2}{*}{0,76} & \multirow{2}{*}{ Đạt } \\
\hline & 706,2 & 112,8 & & & & \\
\hline \multirow{2}{*}{$L N n T$} & 706,9 & $178,9^{*}$ & \multirow{2}{*}{4} & \multirow[t]{2}{*}{0,77} & \multirow[t]{2}{*}{0,75} & \multirow{2}{*}{ Đạt } \\
\hline & 706,9 & 381,9 & & & & \\
\hline \multirow{2}{*}{ 3'-SL } & 632,0 & $290,0^{*}$ & \multirow{2}{*}{4} & \multirow[t]{2}{*}{0,87} & \multirow[t]{2}{*}{0,89} & \multirow{2}{*}{ Đạt } \\
\hline & 632,0 & 86,7 & & & & \\
\hline \multirow{2}{*}{ 6'-SL } & 632,1 & $298,9^{*}$ & \multirow{2}{*}{4} & \multirow[t]{2}{*}{0,90} & \multirow[t]{2}{*}{0,92} & \multirow{2}{*}{ Đạt } \\
\hline & 632,1 & 86,8 & & & & \\
\hline
\end{tabular}


*: Mảnh định lượng

a: Tỷ lệ ion = mảnh định tính/ mảnh định lượng

3.4.2. Giới hạn phát hiện (LOD) và giới hạn định lượng (LOQ)

Dựa trên tỷ số tín hiệu/nhiễu nền của các dung dịch thêm chuẩn, giới hạn phát hiện và giới hạn định lượng của các HMOs thu được trong Bảng 4.

Bảng 4. Kết quả giới hạn phát hiện - Giới hạn định lượng

\begin{tabular}{cccc}
\hline Nền mẫu & Chất phân tích & LOD $(\mathbf{m g} / \mathbf{k g})$ & LOQ $(\mathbf{m g} / \mathbf{k g})$ \\
\hline \multirow{3}{*}{ TPBS dạng bột } & 2'-FL & 4,00 & 10,0 \\
& LNT & 2,00 & 5,00 \\
& LNnT & 2,00 & 5,00 \\
& 3'-SL & 2,00 & 5,00 \\
& 6'SL & 2,00 & 5,00 \\
\cline { 2 - 4 } TPS dạng lỏng & 2'-FL & 4,00 & 10,0 \\
& LNT & 2,00 & 5,00 \\
& LNnT & 2,00 & 5,00 \\
& 3'-SL & 2,00 & 5,00 \\
\hline
\end{tabular}

3.4.3. Độ lặp lại và độ tái lập nội bộ

Thực hiện phân tích mẫu thực chứa 2'-FL, LNT, LNnT, 3'-SL, 6'SL. Phân tích lặp lại 06 lần để xác định độ lặp lại và 10 lần mỗi kiểm nghiệm viên để tính độ tái lập giữa hai kiểm nghiệm viên trong hai ngày khác nhau. Kết quả phân tích độ tái lập nội bộ của phương pháp được trình bày trong Bảng 5 .

Bảng 5. Kết quả phân tích độ tái lập nội bộ

\begin{tabular}{ccccc}
\hline Chất phân tích & Hàm lượng & Múc yêu cầu & Kết quả & Kết luận \\
\hline 2'-FL & $0,1 \%$ & $\leq 8,0 \%$ & $\mathbf{2 , 6 8}$ & Đạt \\
LNT & $100 \mathrm{mg} / \mathrm{kg}$ & $\leq 6,0 \%$ & $\mathbf{3 , 9 7}$ & Đạt \\
LNnT & $100 \mathrm{mg} / \mathrm{kg}$ & $\leq 6,0 \%$ & $\mathbf{2 , 1 4}$ & Đạt \\
3'-SL & $0,1 \%$ & $\leq 8,0 \%$ & $\mathbf{3 , 8 9}$ & Đạt \\
6'-SL & $0,1 \%$ & $\leq 8,0 \%$ & $\mathbf{4 , 1 9}$ & Đạt \\
\hline
\end{tabular}

Kết quả thẩm định cho thấy phương pháp có độ lặp tốt: RSD đạt đáp ứng yêu cầu của AOAC [7].

\subsection{4. Độ thu hồi}

Độ thu hôi được đánh giá khi thêm chuẩn ở 02 mức hàm lượng $0,1 \mathrm{~g} / 100 \mathrm{~g}$ (hoặc $0,1 \mathrm{~g} / 100$ $\mathrm{mL}$ ) và và $100 \mathrm{mg} / \mathrm{kg}(100 \mathrm{mg} / \mathrm{L})$ của 2'-FL, LNT, LNnT, 3'-SL, 6'SL vào nền mẫu TPBS (dạng bột, dạng lỏng). Kết quả độ thu hôi được tóm tắt trong Bảng 6 . 
Bảng 6. Kết quả đánh giá độ thu hổi trên nền TPBS dạng bột và dạng lỏng

\begin{tabular}{|c|c|c|c|c|}
\hline Nền mẫu & Chất phân tích & $\begin{array}{l}\text { Mức hàm } \\
\text { luợng thêm } \\
\text { chuẩn }\end{array}$ & Mức yêu cầu & Kết quả \\
\hline \multirow{10}{*}{ TPBS dạng bột } & $2^{\prime}-F L$ & \multirow{5}{*}{$0,1 \mathrm{~g} / 100 \mathrm{~g}$} & $95-105 \%$ & $98,8-102 \%$ \\
\hline & $L N T$ & & $95-105 \%$ & $98,2-102 \%$ \\
\hline & $L N n T$ & & $95-105 \%$ & $98,0-103 \%$ \\
\hline & 3'-SL & & $95-105 \%$ & $97,5-103 \%$ \\
\hline & 6'-SL & & $95-105 \%$ & $96,8-102 \%$ \\
\hline & $2^{\prime}-F L$ & \multirow{5}{*}{$100 \mathrm{mg} / \mathrm{kg}$} & $90-107 \%$ & $99,4-102 \%$ \\
\hline & $L N T$ & & $90-107 \%$ & $97,6-102 \%$ \\
\hline & $L N n T$ & & $90-107 \%$ & $97,8-102 \%$ \\
\hline & 3'-SL & & $90-107 \%$ & $97,8-103 \%$ \\
\hline & 6'-SL & & $90-107 \%$ & $96,4-102 \%$ \\
\hline \multirow{10}{*}{ TPBS dạng lỏng } & $2^{\prime}-F L$ & \multirow{5}{*}{$0,1 \mathrm{~g} / 100 \mathrm{~mL}$} & $95-105 \%$ & $98,0-101 \%$ \\
\hline & $L N T$ & & $95-105 \%$ & $100-102 \%$ \\
\hline & $L N n T$ & & $95-105 \%$ & $97,0-102 \%$ \\
\hline & 3'-SL & & $95-105 \%$ & $98,7-103 \%$ \\
\hline & 6'-SL & & $95-105 \%$ & $96,2-102 \%$ \\
\hline & $2^{\prime}-F L$ & \multirow{5}{*}{$100 \mathrm{mg} / \mathrm{L}$} & $90-107 \%$ & $96,9-103 \%$ \\
\hline & $L N T$ & & $90-107 \%$ & $97,3-101 \%$ \\
\hline & $L N n T$ & & $90-107 \%$ & $97,8-103 \%$ \\
\hline & 3'-SL & & $90-107 \%$ & $97,9-102 \%$ \\
\hline & 6'-SL & & $90-107 \%$ & $95,5-102 \%$ \\
\hline
\end{tabular}

Kết quả thẩm định cho thấy độ thu hồi đạt theo yêu câu AOAC.

\subsubsection{Kết quả phân tích mẫu thực tế}

Sau khi thẩm định đạt yêu cầu của $\mathrm{AOAC}$, quy trình phân tích đã được áp dụng để phân tích hàm lượng 05 HMOs trong 25 mẫu bao gồm 15 mẫu TPBS dạng bột và 10 mẫu TPBS dạng lỏng. Các mẫu được lựa chọn là các mẫu có công bố trên nhãn chứa thành phân HMOs hoặc 2’-FL. Kết quả phân tích được trình bày trong Bảng 7 và Bảng 8 . 
Bảng 7. Kết quả phân tích 2'-FL, LNT, LNnT, 3'-SL, 6'SL trong mẫu dạng bột

\begin{tabular}{|c|c|c|c|c|c|}
\hline $\begin{array}{l}\text { Ký hiệu } \\
\text { mâau }\end{array}$ & $\begin{array}{c}\text { Hàm lượng } \\
\text { 2'-FL (mg/100g) }\end{array}$ & $\begin{array}{c}\text { Hàm lượng } \\
\text { LNT (mg/100g) }\end{array}$ & $\begin{array}{c}\text { Hàm lượng } \\
\text { LNnT } \\
(m g / 100 g)\end{array}$ & $\begin{array}{l}\text { Hàm lượng 3'- } \\
\text { SL (mg/100g) }\end{array}$ & $\begin{array}{l}\text { Hàm lượng 6'- } \\
\text { SL (mg/100g) }\end{array}$ \\
\hline$S B-1$ & 120 & 23,0 & - & - & - \\
\hline$S B-2$ & 115 & 25,1 & - & - & - \\
\hline$S B-3$ & 256 & 14,8 & - & 0,85 & 0,23 \\
\hline$S B-4$ & 275 & 19,8 & - & 0,63 & 0,25 \\
\hline$S B-5$ & 162 & 10,1 & - & - & - \\
\hline$S B-6$ & 109 & 36,1 & - & 0,15 & 0,62 \\
\hline$S B-7$ & 152 & - & - & 0,54 & 0,45 \\
\hline$S B-8$ & 147 & 14,9 & 0,15 & - & - \\
\hline$S B-9$ & 159 & 41,1 & 0,29 & 0,15 & 0,11 \\
\hline$S B-10$ & 296 & - & 0,95 & - & - \\
\hline$S B-11$ & 243 & 22,5 & - & - & - \\
\hline$S B-12$ & 158 & - & - & - & - \\
\hline$S B-13$ & 255 & - & - & - & - \\
\hline$S B-14$ & 201 & - & - & - & - \\
\hline$S B-15$ & 162 & - & - & - & - \\
\hline
\end{tabular}

Ghi chú: “-“: Không phát hiện

Bảng 8. Kết quả phân tích 2'-FL, LNT, LNnT, 3'-SL, 6'SL trong mẫu dạng lỏng

\begin{tabular}{|c|c|c|c|c|c|}
\hline $\begin{array}{l}\text { Ký hiệu } \\
\text { mẫu }\end{array}$ & $\begin{array}{c}\text { Hàm lượng } \\
\text { 2'-FL }(m g / L)\end{array}$ & $\begin{array}{l}\text { Hàm lượng } \\
\text { LNT (mg/L) }\end{array}$ & $\begin{array}{l}\text { Hàm lượng } \\
\text { LNnT }(m g / L)\end{array}$ & $\begin{array}{c}\text { Hàm lượng 3'- } \\
\text { SL }(m g / L)\end{array}$ & $\begin{array}{l}\text { Hàm lượng 6'- } \\
\text { SL (mg/L) }\end{array}$ \\
\hline$S L-1$ & 22,1 & - & - & - & - \\
\hline$S L-2$ & 15,5 & - & - & - & - \\
\hline$S L-3$ & 21,1 & 1,01 & - & - & - \\
\hline$S L-4$ & 25,7 & 1,12 & - & - & - \\
\hline$S L-5$ & 23,4 & 0,98 & - & - & - \\
\hline$S L-6$ & 19,2 & 1,05 & - & - & - \\
\hline$S L-7$ & 13,8 & - & - & - & - \\
\hline$S L-8$ & 21,7 & - & - & - & - \\
\hline$S L-9$ & 29,6 & - & - & - & - \\
\hline$S L-10$ & 21,5 & - & - & - & - \\
\hline
\end{tabular}

Ghi chú: “-“: Không phát hiện

Như vậy có thể thấy, hiện nay trên thị trường, các sản phẩm TPBS có bổ sung HMOs được công bố trên nhãn chủ yếu tập trung vào 02 loại HMOs chính là 2'-FL và LNT; các HMOs như 3'-SL và 6'-SL hâu như không xuất hiện trong các mẫu thực hoặc chiếm một tỷ lệ rất nhỏ. Tổng hàm lượng HMOs được bổ sung vào nền TPBS dạng bột khoảng $0,1-0,3 \mathrm{~g} / 100 \mathrm{~g}$, trong khi đó hàm lượng này giảm gần 10 lần trong TPBS dạng lỏng với hàm lượng khoảng $0,01-0,03$ $\mathrm{g} / 100 \mathrm{~mL}$. Các kết quả phân tích trên cho thấy, với các sản phẩm có công bố thành phần 2'- FL, 
hàm lượng phân tích phù hợp với công bố trên nhãn sản phẩm, các sản phẩm công bố tổng lượng HMOs đều đạt từ $85 \%$ trở lên so với công bố trên nhãn. Tuy nhiên, hiện nay vẫn chưa có mức hàm lượng khuyến cáo của các chất nhóm HMOs trong các TPBS, chính vì thế việc kiểm soát chất lượng vẫn còn gặp nhiều khó khăn.

\section{KẾT LUẬN}

Phương pháp định lượng đông thời 2'-FL, LNT, LNnT, 3'-SL, 6'SL bằng LC-MS/MS trong nền mẫu thực phẩm bổ sung đã được xây dựng và thẩm định. Phương pháp đã được thẩm định về độ đặc hiệu, khoảng tuyến tính, giới hạn phát hiện, giới hạn định lượng, độ chụm, độ đúng, độ không đảm bảo đo. Kết quả phân tích thực tế cho thấy hầu hết các mẫu phân tích đều có kết quả phù hợp với công bố trên nhãn của nhà sản xuất với hàm lượng 05 chất phân tích trong khoảng $0,1-0,3 \mathrm{~g} / 100 \mathrm{~g}$ trong TPBS dạng bột, $0,01-0,03 \mathrm{~g} / 100 \mathrm{~mL}$ với TPPBS dạng lỏng. Phương pháp xây dựng đạt yêu cầu để áp dụng phân tích hàm lượng 2'-FL, LNT, LNnT, 3'-SL, 6'SL trong thực phẩm bổ sung, có thể tiến hành phân tích tại các phòng thử nghiệm có hệ thống LC-MS/ MS như một phương pháp thường quy.

\section{TÀI LIỆU THAM KHẢO}

[1] American pregnance association, "Breastfeeding Overview". [Online]. Availble: https:// americanpregnancy.org/healthy-pregnancy/breastfeeding/breastfeeding-overviewcopy-70904/ [Aceessed 02/19/2020]

[2]. M. H. Monaco, J. Kim, and S. M. Donovan, "Human Milk: Composition and Nutritional Value," Encyclopedia of Food and Health, vol. 2016, pp. 357-362, 2016.

[3]. M. Li, "Human milk oligosaccharides shorten rotavirus-induced diarrhea and modulate piglet mucosal immunity and colonic microbiota," ISME Journal, vol. 8, no. 8, pp. 16091620, 2004.

[4]. A. L. Morrow, G. M. Ruiz-Palacios, X. Jiang, and D. S. Newburg, "Human-milk glycans that inhibit pathogen binding protect breast-feeding infants against infectious diarrhea," Journal of Nutrition, vol. 135, no. 5, pp. 1304-130, 2005.

[5]. S. S. Leeuwen, "Challenges and Pitfalls in Human Milk Oligosaccharide Analysis," Nutrients, vol. 11, no. 11, pp. 2684, 2019.

[6]. A. S. Christensen, S. H. Skov, S. E. Lendal, and B. H. Hornshoj, "Quantifying the human milk oligosaccharides 2'-fucosyllactose and 3-fucosyllactose in different food applications by high-performance liquid chromatography with refractive index detection," Journal of Food Science, vol. 85, no. 2, pp. 332-339, 2010.

[7]. S. Austin, D. Cuany, J. Michaud, B. Diehl, and Begoña Casado, "Determination of 2区-Fucosyllactose and Lacto-N-neotetraose in Infant Formula," Molecules, vol. 23, no. 10, pp. 2650, 2010.

[8]. S. S. van Leeuwen, "Challenges and Pitfalls in Human Milk Oligosaccharide Analysis," Nutrients, vol. 11, no. 11, pp. 2684, 2019.

[9]. T. R. I. Cataldi, C. Campa, and G. E. De Benedetto, "Carbohydrate analysis by highperformance anion-exchange chromatography with pulsed amperometric detection: The potential is still growing," Analytical and Bioanalytical Chemistry, vol. 368, no. 8, pp. 739$758,2000$.

[10]. Y.Bao, C.Chen, and D. S. Newburg, "Quantification of neutral human milk oligosaccharides by graphitic carbon high-performance liquid chromatography with tandem mass spectrometry", Analytical Biochemistry, vol. 433, no. 1, pp. 28-35, 2013. 


\title{
Method validation for simultaneous quantification of some Human Milk Oligosaccharides (HMOs) in dietary supplements by liquid chromatography tandem mass spectrometry (LC-MS/MS)
}

\author{
Nguyen Thi Hong Ngoc ${ }^{1}$, Mac Thi Thanh Hoa ${ }^{1}$, Tran Hung Son ${ }^{1}$, Ngo Manh Dung ${ }^{2}$ \\ Cao Cong Khanh', Vu Thi Thanh An ${ }^{1}$, Pham Thanh Ha ${ }^{2}$, Le Thi Hong Hoa ${ }^{1}$ \\ ${ }^{1}$ National Institute for Food Control, Hanoi, Vietnam \\ ${ }^{2}$ Hanoi University of Pharmacy, Vietnam
}

\section{Abstract}

The mobile phase system consists of 2 channels: channel A ( $0.1 \%$ formic acid) and channel $B$ (acetonitril) connected to the HILIC column $(3.5 \mu \mathrm{m}, 2.1 \mathrm{~mm} \times 150 \mathrm{~mm})$ and the MS detector. The time analysis is 10 minutes, this method can identify all 5 substances belonging to the group HMOs. The method of simultaneous quantification some of Human Milk Oligosaccharide in dietary supplements by LC-MS/MS is an accurate and effective method to quickly determine the content of 2'-Fucosyllactose (2 '-FL), Lacto-N-neotetraose (LNnT), Lacto-N-tetraose (LNT)3'Siallylactose (3'-SL) and 6'-Siallylactose (6'-SL) in both dietary supplements powder and liquid. The method has been validated to be accurate, fast, effective and has been applied to aralyris of samples on the market showing high applicability in fealyti. The detection limit and quantitative limit for all 5'-FL, LNnT, LNT, 3'-SL, 6'-SL were $4 \mathrm{mg} / \mathrm{kg}$ and $10 \mathrm{mg} / \mathrm{kg}$, respectively. The linear range of the method ranges from $0.4 \mu \mathrm{g} / \mathrm{mL}$ to $40 \mu \mathrm{g} / \mathrm{mL}$. Other validation parameters include the accuracy (R\% 98.8 -103\%); The precision (RSD\% 1.69 - 5.54\%) can meet the requirement of AOAC. The method was applied in practice to analyze 25 supplementary food samples on the market gives the results of analyzing total HMOs in powdered samples about $0.1-0.3 \mathrm{~g} / 100 \mathrm{~g}$ and for liquid samples about $0.01-0.03 \mathrm{~g} / 100 \mathrm{~mL}$.

Keywords: 2'-Fucosyllactose (2'-FL), lacto-N-tetraose (LNT), HMOs; dietary supplement, $L C-M S / M S$. 